J. Lake Sci. (湖泊科学), 2021, 33(1): 74-85

DOI 10. 18307/2021. 0122

(c) 2021 by Journal of Lake Sciences

\title{
基于水华蓝藻固有光学特性的主要类群定量识别方法”
}

\author{
褚 乔 $^{1,2}$, 张壹荁 $^{1,2}$, 张玉超 ${ }^{1,3 * *}$,马荣华 ${ }^{1,3}$, 胡旻琪 ${ }^{1,2}$ \\ (1:中国科学院南京地理与湖泊研究所中国科学院流域地理学重点实验室,南京 210008) \\ (2: 中国科学院大学,北京 100049) \\ (3:淮阴师范学院,江苏区域现代农业与环境保护协同创新中心, 淮安 223300)
}

\begin{abstract}
摘 要: 蓝藻水华是湖泊水体富营养化的重要特征之一,不同水华蓝藻类群形成的水华特征、危害及其治理方法差异显 著. 因此, 如何快速、准确地掌握不同蓝藻类群的时空分布特征成为实施富营养化湖泊污染治理与生态恢复、蓝藻生态灾 害预测预警中一个亟待解决的科学问题. 本研究基于纯藻种实验室培养和室内光学控制实验, 在微囊藻 (Microcystis)、鱼 腥藻 (Dolichospermum)、束丝藻 (Aphanizomenon) 3 种主要水华蓝藻固有光学特性的基础上, 通过甄别不同水华蓝藻的吸 收、散射和后向散射光谱的特征波段, 构建了基于吸收和散射特性的 5 种水华蓝藻类群的非线性最优化定量识别模型, 其 中, 基于 $440 、 620$ 和 $675 \mathrm{~nm} 3$ 个波段吸收的 a-CIM $440,620,675$ 具有较为稳定的定量识别能力; 并基于野外实测光学特性数 据, 实现了巢湖主要水华蓝藻类群的定量监测, 初步分析了巢湖主要水华蓝藻类群的时空分布特性. 研究表明, 巢湖的水 华蓝藻以鱼腥藻、微囊藻为主, 束丝藻较少, 鱼腥藻主要出现在温度较低的季节, 微囊藻在夏季的西部湖区占优势; 巢湖 水华主要为微囊藻藻华和鱼腥藻藻华, 且浓度较高的蓝藻主要存在于水体表面以下 $20 \mathrm{~cm}$ 范围内; 微囊藻和鱼腥藻在非 藻华断面垂向上均匀分布. 本研究可为富营养化湖泊蓝藻水华预测预警以及相关管理部门决策提供重要的理论依据和 科学支撑.
\end{abstract}

关键词: 富营养化湖泊;水华蓝藻类群;固有光学特性;非线性最优化模型;巢湖

\section{Quantitative identification methods of bloom-forming cyanobacterial groups of inland lakes based on inherent optical properties*}

\author{
Chu Qiao ${ }^{1,2}$, Zhang Yixuan ${ }^{1,2}$, Zhang Yuchao ${ }^{1,3 * *}$, Ma Ronghua ${ }^{1,3} \&$ Hu Minqi ${ }^{1,2}$ \\ (1: Key Laboratory of Watershed Geographic Sciences, Nanjing Institute of Geography and Limnology, Chinese Academy of \\ Sciences, Nanjing 210008, P.R.China) \\ (2: University of Chinese Academy of Sciences, Beijing 100049, P.R.China) \\ ( 3: Jiangsu Collaborative Innovation Center of Regional Modern Agriculture \&Environmental Protection, Huaiyin Normal Uni- \\ versity, Huai'an 223300 , P.R.China)
}

\begin{abstract}
Cyanobacteria bloom is one of the important characteristics of eutrophication in lake waters. The characteristics, hazards, and treatment methods of different bloom-forming cyanobacterial groups are significantly different. Therefore, in the process of implementing eutrophic lake pollution control, ecological restoration, and cyanobacteria ecological disaster prediction and early warning, how to quickly and accurately grasp the spatiotemporal distribution characteristics of different cyanobacteria groups have become an urgent scientific question. This study is based on the laboratory cultivation of pure algae species and indoor optical control experiments. Based on the inherent optical characteristics of the three main bloom-forming cyanobacterial groups: Microcystis, Dolichospermum, and Aphanizomenon, we screened the characteristic bands of the absorption and scattering spectra of different cyanobacterial groups and five nonlinear optimization quantitative identification models were constructed respectively. The model a-CIM $440,620,675$ based on the absorption characteristic bands of 440,620 and $675 \mathrm{~nm}$ shows the best performances. By applying field measured optical characteristics data to this model, quantitative monitoring of the main cyanobacterial group in Lake Chaohu
\end{abstract}

* 2020-01-19 收稿;2020-04-22 收修改稿.

国家自然科学基金项目 (41671371) 和江苏省科技厅社会发展面上项目 (BE2019774) 联合资助.

** 通信作者;E-mail:yczhang@ niglas.ac.cn. 
was achieved, and the temporal and spatial distribution of the main bloom-forming cyanobacterial group in Lake Chaohu was analyzed. The results show that the cyanobacterial groups in Lake Chaohu are dominated by species of Microcystis and Dolichospermum. Microcystis generally occupies the western region of the lake in summer, whereas Dolichospermum dominated in cold seasons. In Lake Chaohu both of Microcystis bloom and Dolichospermum bloom are found in May. The non-algal bloom sections are mainly Microcystis and Dolichospermum, and they are both vertically and uniformly distributed. There are Microcystis and Dolichospermum in Lake Chaohu, and the higher concentration of cyanobacteria mainly exists below the water surface within $20 \mathrm{~cm}$. This study can provide important theoretical basis and scientific support for the prediction and early warning of cyanobacteria blooms in eutrophic lakes and help with the decisions of relevant management departments.

Keywords: Eutrophic lakes; bloom-forming cyanobacteria; inherent optical properties; nonlinear optimization model; Lake Chaohu

我国五大淡水湖中太湖、巢湖、洪泽湖和滇池均处于轻度乃至中度富营养化状态 ${ }^{[1]}$. 蓝藻通常为富营养 化水体的优势藻种 ${ }^{[2-3]}$. 蓝藻的大量繁殖使水体呈现绿色或蓝色, 这是湖泊水体富营养化的重要特征, 水体 蓝藻暴发不仅会造成鱼虾死亡, 导致水体污染, 还会直接威胁到当地经济发展, 我国已经成为世界上蓝藻水 华暴发最严重、分布最广的国家之一 ${ }^{[4]}$. 我国富营养化湖泊中, 形成水华的蓝藻主要包括微囊藻 (Microcystis)、鱼腥藻 (Dolichospermum)、束丝藻 (Aphanizomenon) 和颤藻 (Oscillatoria) 等, 其中以铜绿微囊藻 (M. aeruginosa) 在数量和发生频率上占绝对优势 ${ }^{[5]}$. 根据文献显示, 就年平均水平的群落组成和空间分布而 言, 蓝藻占巢湖藻种的绝对主导地位 (超过总数的 $99.4 \%)^{[6]}$, 且巢湖的蓝藻以微囊藻和鱼腥藻为主, 部分区 域以束丝藻为主 ${ }^{[7]}$. 不同蓝藻类群的危害、环境适应性、时空分布特征以及应急治理方法不同, 因此在实施 富营养化湖泊生态环境治理与恢复、蓝藻生态灾害预测预警的过程中, 掌握不同蓝藻的时空分布特征就显 得尤为重要.

藻类种群识别涉及生物学、光谱分析等多个学科领域, 主要分为传统识别方法和遥感识别方法. 传统藻 类识别方法大都是利用藻类的细胞形态特征 ${ }^{[8-9]}$ 、藻种 $\mathrm{DNA}^{[10]}$ 、色素特征光谱 (高效液相色谱法 ${ }^{[11-12]}$ )、菼光

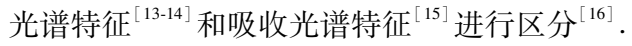

而藻类的遥感识别方法主要依据固有光学特性或者表观光学特性. 藻类的吸收特性和散射特性是目前基 于固有光学特性开展藻类遥感监测的基础. Mahoney ${ }^{[17]}$ 在固有光学特性的基础上建立了腰鞭毛藻的生物光学 定量反演模型; Martinez-Guijarro 等 ${ }^{[18]}$ 根据浮游藻类吸收系数光谱曲线识别浮游藻类组成成分; 黄昌春等 ${ }^{[19]}$ 利 用四阶微分、标准化系数等方法对太湖水体藻类组成进行计算分析. Boddy 等 ${ }^{[20]}$ 利用浮游藻类的苂光特性和 散射特性的差异识别藻类; 马万泉 ${ }^{[21]}$ 、戴红亮 ${ }^{[22]}$ 、吕恒等 ${ }^{[23-24]}$ 和王瑜 ${ }^{[25]}$ 基于室内控制试验及模拟试验, 及色素 吸收特征、藻细胞特征和反射光谱特征等探索性地建立了藻种门类间比例遥感估算方法. 国内外亦有基于藻 类反射光谱特征开展藻类遥感识别的相关研究. 崔延伟等 ${ }^{[26]}$ 利用 $687 \sim 728 \mathrm{~nm}$ 波段的特征反射峰、Lee 等 ${ }^{[27]}$ 利用活体藻类的苂光特性、Hunter 等 ${ }^{[28]}$ 利用不同藻种的反射率光谱, 先后对藻类进行了识别.

由此可见, 目前研究主要集中在海洋中不同藻种的遥感反演, 尽管内陆湖泊亦有少量针对不同门类间 藻类遥感反演的研究报道, 但是针对主要水华蓝藻类群(微囊藻、鱼腥藻、束丝藻)的遥感反演基础理论方面 的研究尚未见到公开发表文献. 张壹萱等 ${ }^{[29]}$ 基于室内控制实验, 对微囊藻、鱼腥藻、束丝藻 3 种典型水华蓝 藻的固有光学特性和影响因子进行研究, 结果表明, 3 种主要水华蓝藻吸收系数的波谱特征趋势相近, 440、 620 和 $675 \mathrm{~nm}$ 波长附近有明显的吸收峰. 鱼腥藻在 $440 \mathrm{~nm}$ 处有较高的吸收能力, 微囊藻在 620 和 $675 \mathrm{~nm}$ 波 长处有较高的吸收能力. 微囊藻与束丝藻散射光谱曲线均呈现随波长增加而下降的趋势, 鱼腥藻的散射波 谱相对缓和且平滑, 3 种水华蓝藻均在 440 和 $675 \mathrm{~nm}$ 处有微弱的散射谷. 这 3 种蓝藻的吸收系数和散射系 数都随着色素浓度 (叶绿素 $a($ Chl. $a$ ) 或藻蓝素) 增加而增大.

本研究在该研究结果 ${ }^{[29]}$ 的基础上,通过甄别不同水华蓝藻的吸收和散射的特征波段,构建微囊藻、鱼腥 藻和束丝藻 3 种水华蓝藻类群的定量识别模型, 并以巢湖为应用区域, 实现主要水华蓝藻类群的定量监测, 为我国富营养化湖泊蓝藻水华的预测、预警提供重要的技术支撑. 


\section{1 实验部分}

\section{1 实验参数测量}

1.1.1 室内实验 本研究在光照培养箱中对铜绿微囊藻、水华鱼腥藻和水华束丝藻 3 种蓝藻进行纯藻种和混合 藻种培养, 培养基均为 BG-11. 经过无菌实验室内的保种, 将纯藻接种至 $50 \mathrm{~mL}$ 的三角雉形瓶中, 生长 $7 \sim 10 \mathrm{~d}$ 后进行逐级扩大培养. 培养环境为温度 $25^{\circ} \mathrm{C}$, 光照强度 $2000 \mathrm{~lx}$, 光周期为 $12 \mathrm{~h}$ 光照 $/ 12 \mathrm{~h}$ 黑暗, 主要流程为: 将 $50 \mathrm{~mL}$ 的三角雉形瓶和配置的营养液进行高温高压灭菌后, 在无菌室进行接种和保种, 每日摇晃 $3 \sim 4$ 次, 生长 7 10 d 后, 重复上述操作扩大培养至 $100 \mathrm{~mL}$, 直至总藻量达到 $5 \mathrm{~L}$, 在藻类生长的稳定期时获取数据.

单藻的室内光学控制实验过程如下: 向有机玻璃桶中加人 $150 \mathrm{~L}$ 纯水和 $2 \mathrm{~L}$ 纯藻液, 后以 $500 \mathrm{~mL}$ 为梯 度分 5 次加人藻的母液, 最终使桶内有 $4.5 \mathrm{~L}$ 藻的母液. 由于束丝藻生长缓慢, 束丝藻实验中以 $500 \mathrm{~mL}$ 为梯 度分 3 次加人藻的母液. 本研究的实验是连续性实验, 所以单藻混合实验的具体过程请参见第一阶段 论文 ${ }^{[29]}$.

双藻混合实验过程如图 $1 \mathrm{a}$ 所示, 向桶中加人 $150 \mathrm{~L}$ 纯水和 $2 \mathrm{~L}$ 纯藻液 $i$, 后以 $500 \mathrm{~mL}$ 为梯度加人纯藻 液 $j$, 测量各参数, 重复操作 4 次, 使 $j$ 藻量最终达到 $2 \mathrm{~L}$ ( $i$ 和 $j$ 分别为微囊藻、鱼腥藻、束丝藻中任意两种), 一共构建 12 组混合实验. 三藻混合实验与单藻和双藻实验类似, 加人 $150 \mathrm{~L}$ 纯水后, 以 $500 \mathrm{~mL}$ 为梯度依次 加人束丝藻、微囊藻、鱼腥藻 $500 \mathrm{~mL}$, 一共构建 10 组混合藻比例 ( 图 $1 \mathrm{~b}$ ).

(a)

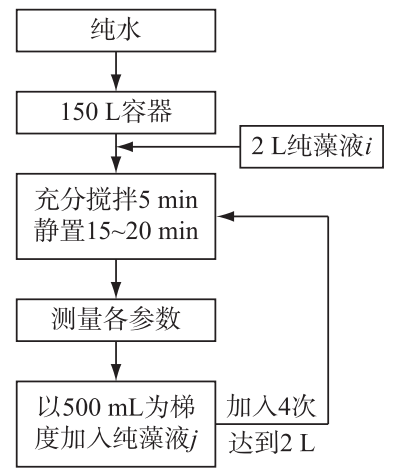

(注: $i$ 和 $j$ 分别为微囊藻、鱼腥藻、束丝藻中任意两种)

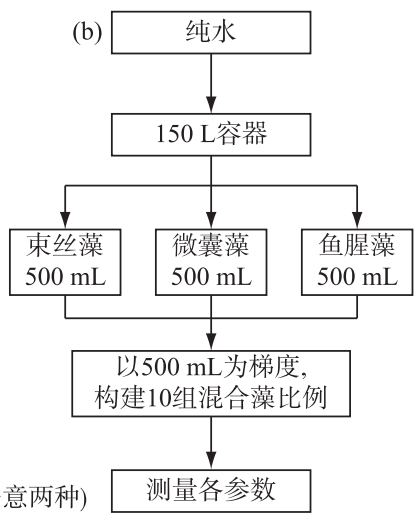

测量各参数

图 1 藻类混合实验流程图: (a) 双藻; (b) 三藻

Fig.1 Experimental flow chart: (a) two cyanobacteria groups; (b) three cyanobacteria groups

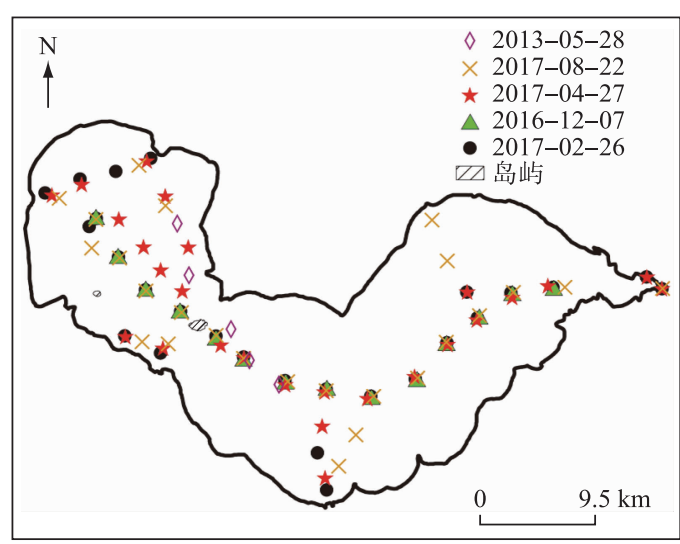

图 2 巢湖野外采样点位分布

Fig. 2 Distribution of sampling sites in Lake Chaohu
1.1 .2 室外实验 巢湖是中国第 5 大淡水湖, 是位于 安徽省中部的富营养化浅水湖泊, 面积 $770 \mathrm{~km}^{2}$ $\left(31^{\circ} 25^{\prime} \sim 31^{\circ} 43^{\prime} \mathrm{N}, 117^{\circ} 17^{\prime} \sim 117^{\circ} 51^{\prime} \mathrm{E}\right.$, 图 2), 平均水 深 $3.0 \mathrm{~m}^{[30]}$. 巢湖富营养化程度严重, 近几十年来, 蓝 藻水华一直是巢湖最严重的环境污染问题之一, 不 仅会引起水质恶化, 破坏生态系统结构, 还会引起水 体生态系统功能改退化, 造成生态环境风险或直接 的环境污染. 因此, 开展巢湖蓝藻类群的识别研究对 于评价蓝藻生态环境风险、有针对性地采取措施控 制巢湖水华蓝藻以及建立水质的预警系统尤为重要.

2013 年 5 月进行 1 次巢湖野外垂向采样, 2016 年 12 月- 2017 年 8 月对巢湖进行了 4 次表层巡测采 样(图 2). 垂向采样每个点位采集 9 层水样, 包括表 层、深度 $0.1 、 0.2 、 0.4 、 0.7 、 1.0 、 1.5 、 2.0$ 和 $3.0 \mathrm{~m}$. 垂向 
分层水样采集使用自制的垂向水样采集器, 主要由直径 $10 \mathrm{~cm}$ 的抽水洜、连接管、标尺,进水口的位置和深 度参考标尺组成. 表层水样直接用采水瓶采集. 现场采集的水样放在冷藏保温箱里遮光保存, 当天进行过滤 处理, 实验室内实测数据包括 Chl. $a$ 浓度及其对应组分的吸收系数.

1.1.3 参数测量 颗粒物吸收系数采用定量滤膜技术测定. 用分光光度计 (UV-2600) 测量过滤水样后直径为 $47 \mathrm{~mm}$ 的 $\mathrm{GF} / \mathrm{F}$ 膜得到总颗粒物吸收系数 $\left(a_{\mathrm{p}}\right)$; 再用 $\mathrm{NaClO}_{3}$ 对滤膜上的总悬浮颗粒物进行漂白, 从而测得 非色素颗粒物吸收系数 $\left(a_{\mathrm{d}}\right)$; 浮游植物色素吸收系数 $\left(a_{\mathrm{ph}}\right)$ 为 $a_{\mathrm{p}}$ 与 $a_{\mathrm{d}}$ 的差值 ${ }^{[31]}$.

Chl. $a$ 浓度采用丙酮萃取法及分光光度计法测定. 用 $1.2 \mu \mathrm{m}$ 的 $\mathrm{GF} / \mathrm{C}$ 滤膜过滤水样,使用液氮反复冻融 3 次破碎藻细胞,加人 $90 \%$ 丙酮溶液后经过离心机 ( TG16-WS) 离心提取 Chl. $a$, 而后用分光光度计分别在 $750 、 663 、 645$ 和 $630 \mathrm{~nm}$ 波长处测定叶绿素吸光度值, 从而获得 Chl. $a$ 的浓度 ${ }^{[32-34]}$.

吸收和衰减系数测量: 光学控制实验中使用的仪器为 Wetlabs 公司的高光谱吸收/衰减仪 (AC-S). 该仪 器在 $400 \sim 730 \mathrm{~nm}$ 光谱范围内有 80 个波段输出, 光谱分辨率为 $4 \mathrm{~nm}$, 测量精准度为 $\pm 0.01 \mathrm{~m}^{-1}$. 该仪器可以 同步测量吸收和衰减系数,能够通过保证同步性来控制实验误差.

\section{2 水华蓝藻类群定量识别模型构建}

浮游藻类的吸收、散射以及后向散射特性均属于固有光学特性, 具有严格的累加性 ${ }^{[35]}$, 因此, 混合蓝藻 的固有光学特性是每个组成蓝藻固有光学特性的线性叠加, 因此, 本研究中 3 种水华蓝藻的总吸收系数和 总散射系数可以转化为每种蓝藻的吸收系数和散射系数的多元线性回归. 由于本研究中水华蓝藻主要为 3

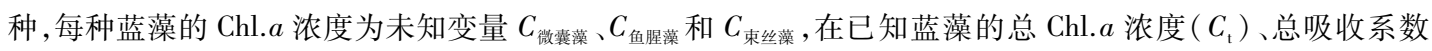

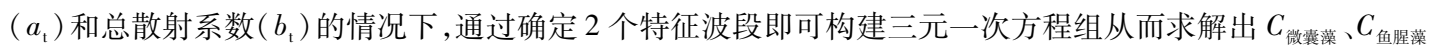
和 $C_{\text {束丝莱. }}$.

基于固有光学特性分析 ${ }^{[29]}$,可用于定量识别 3 种水华蓝藻固有光学特性的特征波段汇总如表 1 所示. 本研究依据这些特征波段,分别构建基于 吸收特征波段、散射特征波段以及吸收散射特征 波段的定量识别模型.

1.2.1 单位叶绿素 $a$ 浓度的固有光学特性曲线拟 合 受藻密度和包裹效应的影响, 单位 Chl. $a$ 浓 度藻类的吸收、散射系数并不固定,因此需要通 过实验与公式拟合来建立与 Chl. $a$ 浓度的关系，

表 1 主要水华蓝藻固有光学特性的特征波段

Tab.1 Feature bands of inherent optical properties of main bloom-forming cyanobacterial groups

\begin{tabular}{ccc}
\hline 固有光学特性 特征波段/nm & 原理 \\
\hline 吸收特性 & 440 & 叶绿素 $a$ 及其他色素吸收峰 \\
& 620 & 藻蓝素吸收峰 \\
& 675 & 叶绿素 $a$ 吸收峰 \\
散射特性 & 440 & 散射谷 \\
& 675 & 散射谷 \\
\hline
\end{tabular}
从而去除包裹效应的影响. 室内光学实验的结果 显示: 单位 Chl. $a$ 浓度藻类的吸收以及散射光谱与相关研究具有较好的一致性 ${ }^{[29]}$, 在此基础上本研究分别 拟合了 3 种水华蓝藻在特征波段上的吸收、散射系数随 Chl. $a$ 浓度变化的曲线,即:

$$
\begin{aligned}
a_{i, \lambda_{a}}^{*} & =f_{\mathrm{a}, i}\left(C_{i}\right) \\
b_{i, \lambda_{\mathrm{v}}}^{*} & =f_{\mathrm{b}, i}\left(C_{i}\right)
\end{aligned}
$$

式中, $a^{*} 、 b^{*}$ 分别为单位 Chl. $a$ 浓度藻类的吸收、散射系数, $\mathrm{m}^{2} / \mathrm{mg} ; f_{\mathrm{a}} f_{\mathrm{b}}$ 分别为拟合函数; $C$ 为 Chl. $a$ 浓度, $\mathrm{mg} / \mathrm{m}^{3} ; i$ 为微囊藻、鱼腥藻或束丝藻; $\lambda_{\mathrm{a}} 、 \lambda_{\mathrm{b}}$ 分别为吸收、散射的特征波段, $\mathrm{nm} .3$ 种蓝藻在特征波段上,单位 Chl. $a$ 浓度固有光学特性拟合曲线详见表 2 .

每种蓝藻的吸收、散射将通过该浓度对应的单位吸收、散射系数进行计算,即:

式中, $a 、 b$ 分别为吸收、散射系数, $\mathrm{m}^{-1}$.

$$
\begin{aligned}
a_{i, \lambda_{\mathrm{s}}} & =C_{i} \cdot a_{i, \lambda_{s}}^{*} \\
b_{i, \lambda_{\mathrm{b}}} & =C_{i} \cdot b_{i, \lambda_{\mathrm{w}}}^{*}
\end{aligned}
$$

1.2.2 基于固有光学特性的水华蓝藻类群定量识别模型 1 ) 模型构建: 固有光学特性和 Chl. $a$ 浓度均具有加 和性, 因此, 本研究中蓝藻的 $a_{\mathrm{t}}$ 即为 $a_{\mathrm{m}} 、 a_{\mathrm{d}}$ 和 $a_{\mathrm{a}}$ 吸收系数之和, $C_{\mathrm{t}}$ 亦为 3 种蓝藻的浓度 $\left(C_{\mathrm{m}} 、 C_{\mathrm{d}} 、 C_{\mathrm{a}}\right)$ 之 和, 即: 


$$
\begin{gathered}
a_{\mathrm{t}}(\lambda)=a_{\mathrm{m}}(\lambda)+a_{\mathrm{d}}(\lambda)+a_{\mathrm{a}}(\lambda) \\
C_{\mathrm{t}}=C_{\mathrm{m}}+C_{\mathrm{d}}+C_{\mathrm{a}} \\
\lambda=440,620,675 \mathrm{~nm}
\end{gathered}
$$

\begin{tabular}{|c|c|c|}
\hline 类型 & 特征波段 $\lambda / \mathrm{nm}$ & $f_{\mathrm{m}}$ \\
\hline \multirow[t]{3}{*}{$a^{*}$} & 440 & $a_{\mathrm{m}, 440}^{*}=0.0619 C_{\mathrm{m}}^{-0.194}$ \\
\hline & 620 & $a_{\mathrm{m}, 620}^{*}=0.033 C_{\mathrm{m}}^{-0.198}$ \\
\hline & 675 & $a_{\mathrm{m}, 675}^{*}=0.0312 C_{\mathrm{m}}^{-0.195}$ \\
\hline \multirow[t]{2}{*}{$b^{*}$} & 440 & $b_{\mathrm{m}, 440}^{*}=0.1579 C_{\mathrm{m}}^{-0.162}$ \\
\hline & 675 & $b_{\mathrm{m}, 675}^{*}=0.108 C_{\mathrm{m}}^{-0.134}$ \\
\hline 类型 & 特征波段 $\lambda / \mathrm{nm}$ & $f_{\mathrm{d}}$ \\
\hline \multirow[t]{3}{*}{$a^{*}$} & 440 & $a_{\mathrm{d}, 440}^{*}=0.0777 C_{\mathrm{d}}^{-0.237}$ \\
\hline & 620 & $a_{\mathrm{d}, 620}^{*}=0.0324 C_{\mathrm{d}}^{-0.265}$ \\
\hline & 675 & $a_{\mathrm{d}, 675}^{*}=0.0337 C_{\mathrm{d}}^{-0.235}$ \\
\hline \multirow[t]{2}{*}{$b^{*}$} & 440 & $b_{d, 440}^{*}=0.2007 C_{\mathrm{d}}^{-0.194}$ \\
\hline & 675 & $b_{\mathrm{d}, 675}^{*}=0.2386 C_{\mathrm{d}}^{-0.229}$ \\
\hline 类型 & 特征波段 $\lambda / \mathrm{nm}$ & $f_{\mathrm{a}}$ \\
\hline \multirow[t]{3}{*}{$a^{*}$} & 440 & $a_{\mathrm{a}, 440}^{*}=0.0613 C_{\mathrm{a}}^{-0.198}$ \\
\hline & 620 & $a_{\mathrm{a}, 620}^{*}=0.024 C_{\mathrm{a}}^{-0.173}$ \\
\hline & 675 & $a_{\mathrm{a}, 675}^{*}=0.023 C_{\mathrm{a}}^{-0.174}$ \\
\hline \multirow[t]{2}{*}{$b^{*}$} & 440 & $b_{a, 440}^{*}=0.504 C_{\mathrm{a}}^{-0.208}$ \\
\hline & 675 & $b_{\mathrm{a}, 675}^{*}=0.3097 C_{\mathrm{a}}^{-0.205}$ \\
\hline
\end{tabular}

表 2 单位叶绿素 $a$ 浓度的固有光学特性与叶绿素 $a$ 浓度间的拟合曲线汇总

Tab.2 Fitting curves of specific inherent optical properties and chlorophyll- $a$ concentrations

结合公式 (1) 和 (3), 将蓝藻在 2 个特征波段总吸收的加和性作为限制条件, 总 Chl. $a$ 浓度的加和性作 为目标函数, 可采用线性最优规划方法求解 3 种蓝藻的 Chl. $a$ 浓度值.

本研究建立的基于吸收特性的水华蓝藻类群定量识别模型如下:

a-CIM $_{440,620}$ :

$$
\begin{gathered}
\min Z=\left|C_{\mathrm{t}}-\left(C_{\mathrm{m}}+C_{\mathrm{d}}+C_{\mathrm{a}}\right)\right| \\
\text { s.t. } a_{\mathrm{t}}(440)=C_{\mathrm{m}} \cdot a_{\mathrm{m}, 440}^{*}+C_{\mathrm{d}} \cdot a_{\mathrm{d}, 440}^{*}+C_{\mathrm{a}} \cdot a_{\mathrm{a}, 440}^{*} \\
a_{\mathrm{t}}(620)=C_{\mathrm{m}} \cdot a_{\mathrm{m}, 620}^{*}+C_{\mathrm{d}} \cdot a_{\mathrm{d}, 620}^{*}+C_{\mathrm{a}} \cdot a_{\mathrm{a}, 620}^{*} \\
C_{\mathrm{m}}, C_{\mathrm{d}}, C_{\mathrm{a}} \geqslant 0
\end{gathered}
$$

a-CIM $_{440,675}$ :

$$
\begin{gathered}
\min Z=\left|C_{\mathrm{t}}-\left(C_{\mathrm{m}}+C_{\mathrm{d}}+C_{\mathrm{a}}\right)\right| \\
\text { s.t. } a_{\mathrm{t}}(440)=C_{\mathrm{m}} \cdot a_{\mathrm{m}, 440}^{*}+C_{\mathrm{d}} \cdot a_{\mathrm{d}, 440}^{*}+C_{\mathrm{a}} \cdot a_{\mathrm{a}, 440}^{*} \\
a_{\mathrm{t}}(675)=C_{\mathrm{m}} \cdot a_{\mathrm{m}, 675}^{*}+C_{\mathrm{d}} \cdot a_{\mathrm{d}, 675}^{*}+C_{\mathrm{a}} \cdot a_{\mathrm{a}, 675}^{*} \\
C_{\mathrm{m}}, C_{\mathrm{d}}, C_{\mathrm{a}} \geqslant 0
\end{gathered}
$$

a-CIM $_{620,675}$ :

$$
\begin{gathered}
\min Z=\left|C_{\mathrm{t}}-\left(C_{\mathrm{m}}+C_{\mathrm{d}}+C_{\mathrm{a}}\right)\right| \\
\text { s.t. } a_{\mathrm{t}}(620)=C_{\mathrm{m}} \cdot a_{\mathrm{m}, 620}^{*}+C_{\mathrm{d}} \cdot a_{\mathrm{d}, 620}^{*}+C_{\mathrm{a}} \cdot a_{\mathrm{a}, 620}^{*} \\
a_{\mathrm{t}}(675)=C_{\mathrm{m}} \cdot a_{\mathrm{m}, 675}^{*}+C_{\mathrm{d}} \cdot a_{\mathrm{d}, 675}^{*}+C_{\mathrm{a}} \cdot a_{\mathrm{a}, 675}^{*} \\
C_{\mathrm{m}}, C_{\mathrm{d}}, C_{\mathrm{a}} \geqslant 0
\end{gathered}
$$

a-CIM $_{440,620,675}$ : 


$$
\begin{gathered}
\min Z=\left|C_{\mathrm{t}}-\left(C_{\mathrm{m}}+C_{\mathrm{d}}+C_{\mathrm{a}}\right)\right| \\
\text { s.t. } a_{\mathrm{t}}(440)=C_{\mathrm{m}} \cdot a_{\mathrm{m}, 440}^{*}+C_{\mathrm{d}} \cdot a_{\mathrm{d}, 440}^{*}+C_{\mathrm{a}} \cdot a_{\mathrm{a}, 440}^{*} \\
a_{\mathrm{t}}(620)=C_{\mathrm{m}} \cdot a_{\mathrm{m}, 620}^{*}+C_{\mathrm{d}} \cdot a_{\mathrm{d}, 620}^{*}+C_{\mathrm{a}} \cdot a_{\mathrm{a}, 620}^{*} \\
a_{\mathrm{t}}(675)=C_{\mathrm{m}} \cdot a_{\mathrm{m}, 675}^{*}+C_{\mathrm{d}} \cdot a_{\mathrm{d}, 675}^{*}+C_{\mathrm{a}} \cdot a_{\mathrm{a}, 675}^{*} \\
C_{\mathrm{m}}, C_{\mathrm{d}}, C_{\mathrm{a}} \geqslant 0
\end{gathered}
$$

与吸收特性类似, 蓝藻的 $b_{\mathrm{t}}$ 为其所包含 3 种水华蓝藻散射系数 $\left(b_{\mathrm{m}} 、 b_{\mathrm{d}} 、 b_{\mathrm{a}}\right)$ 之和, $C_{\mathrm{t}}$ 亦为 3 种蓝藻的浓 度 $\left(C_{\mathrm{m}} 、 C_{\mathrm{d}} 、 C_{\mathrm{a}}\right)$ 之和, 即:

$$
\begin{gathered}
b_{\mathrm{t}}(\lambda)=b_{\mathrm{m}}(\lambda)+b_{\mathrm{d}}(\lambda)+b_{\mathrm{a}}(\lambda) \\
C_{\mathrm{t}}=C_{\mathrm{m}}+C_{\mathrm{d}}+C_{\mathrm{a}} \\
\lambda=440,675 \mathrm{~nm}
\end{gathered}
$$

本研究中, 结合公式 (2) 和 (4), 同样依据类似的方法采用线性最优规划方法求解 3 种蓝藻的 Chl. $a$ 浓 度值.

基于散射特性的水华蓝藻类群定量识别模型如下:

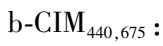

$$
\begin{gathered}
\min Z=\left|C_{\mathrm{t}}-\left(C_{\mathrm{m}}+C_{\mathrm{d}}+C_{\mathrm{a}}\right)\right| \\
\text { s.t. } b_{\mathrm{t}}(440)=C_{\mathrm{m}} \cdot b_{\mathrm{m}, 440}^{*}+C_{\mathrm{d}} \cdot b_{\mathrm{d}, 440}^{*}+C_{\mathrm{a}} \cdot b_{\mathrm{a}, 440}^{*} \\
b_{t}(675)=C_{\mathrm{m}} \cdot b_{\mathrm{m}, 675}^{*}+C_{\mathrm{d}} \cdot b_{\mathrm{d}, 675}^{*}+C_{\mathrm{a}} \cdot b_{\mathrm{a}, 675}^{*} \\
C_{\mathrm{m}}, C_{\mathrm{d}}, C_{\mathrm{a}} \geqslant 0
\end{gathered}
$$

2) 精度评价方法: 上述水华蓝藻类群定量识别模型采用非线性最优化方法求得最终结果, 具体实施基 于 MATLAB 语言中 fmincon ( ) 函数, 主要分为目标函数和限制条件两部分, 目标函数是 Chl. $a$ 总浓度的加和 性, 限制条件具体指 Chl. $a$ 浓度与 3 种蓝藻固有光学特性间的拟合公式, 在此基础上同时定义 3 种蓝藻的 Chl. $a$ 浓度介于 0 和总浓度之间,通过编程实现非线性多元函数求最优解的计算.

为了评价模型的精度, 本研究将双藻和三藻混合实验数据作为验证数据, 同时选取均方根误差 $(R M S E)$ 、相对均方根误差 $\left(R M S E_{\mathrm{rel}}\right)$ 、平均标准差 $(M N B)$ 以及标准均方根误差 $(N R M S)^{[36]}$ 作为模型精度评 价指标:

$$
\begin{aligned}
& \alpha_{i}=Y_{i}-X_{i} \\
& \beta_{i}=\frac{Y_{i}-X_{i}}{X_{i}} \\
& R M S E=\sqrt{\frac{1}{N} \sum_{i=1}^{N} \alpha_{i}^{2}} \\
& R M S E_{\mathrm{rel}}=\sqrt{\frac{1}{N} \sum_{i=1}^{N} \beta_{i}^{2}} \\
& M N B=\frac{1}{N} \sum_{i=1}^{N} \beta_{i} \\
& N R M S=\sqrt{\frac{1}{N} \sum_{i=1}^{N}\left(\beta_{i}-M N B\right)^{2}}
\end{aligned}
$$

式中, $X$ 为实测值, $Y$ 为模型计算值.

\section{2 结果与讨论}

\section{1 藻类实验结果}

基于上述评价指标, 本研究对所构建的 5 种蓝藻类群水华定量识别模型分别进行了精度评价, 具体结 果如表 3 所示.

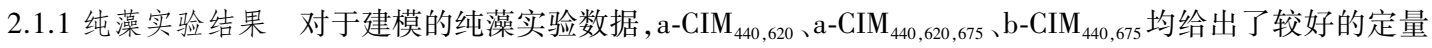


识别结果. 3 种主要水华蓝藻在 $440 、 620$ 和 $675 \mathrm{~nm}$ 处的吸收和散射特征差异显著, 因此在互不干扰的情况 下, 上述 3 种模型对 3 种水华蓝藻的定量识别能力较高. 图 3a 展示了基于 a-CIM ${ }_{440,620,675}$ 的不同蓝藻类群定 量识别结果和监测值的对比. 由该图可知, a-CIM $440,620,675$ 模型对微囊藻和鱼腥藻的识别精度较高, 图中点位 基本分布在 $1: 1$ 线附近,而束丝藻则在高浓度区域出现了一定程度的低估.

2.1 .2 双藻实验结果 双藻混合实验数据中所有模型的定量识别能力都有所下降, 总体上, 基于吸收的模型 的定量识别能力明显优于基于散射的模型, 其中基于 $440 、 620$ 和 $675 \mathrm{~nm} 3$ 个波段吸收的 a-CIM ${ }_{440,620,675}$ 显示 了较为稳定的定量识别能力, 而基于两个波段吸收的模型定量识别能力较弱, 说明混合藻类实验中, 不同蓝 藻之间有所干扰, 因此从混合蓝藻中定量识别不同蓝藻需要更多波段信息才更准确. 图 $3 \mathrm{~b}$ 是基于双藻混合 实验数据 a-CIM $440,620,675$ 模型计算值与监测值的对比, 由该图可知, a-CIM 4 $_{40,620,675}$ 模型对微囊藻和鱼腥藻的定 量识别结果与监测值较好地分布在 $1: 1$ 线附近, 而对束丝藻的定量识别结果略低于监测值.

表 3 模型评价结果

Tab.3 Model assessment results

\begin{tabular}{|c|c|c|c|c|c|}
\hline \multirow{2}{*}{$\begin{array}{l}\text { 评价 } \\
\text { 指标 }\end{array}$} & \multicolumn{5}{|c|}{ 纯藻实验 } \\
\hline & ${\mathrm{a}-\mathrm{CIM}_{440,620}}_{2}$ & 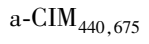 & 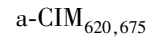 & a-CIM $440,620,675$ & b-CIM ${ }_{440,675}$ \\
\hline RMSE & 4.0385 & 9.5791 & 15.4272 & 4.2591 & 4.3682 \\
\hline$R M S E_{\text {rel }}$ & 0.1254 & 0.3567 & 0.4592 & 0.1212 & 0.1695 \\
\hline$M N B$ & -0.0901 & -0.2118 & -0.3297 & -0.0848 & -0.0899 \\
\hline NRMS & 0.0901 & 0.2964 & 0.3302 & 0.0895 & 0.1484 \\
\hline \multirow{2}{*}{$\begin{array}{l}\text { 评价 } \\
\text { 指标 }\end{array}$} & \multicolumn{5}{|c|}{ 双藻混合实验 } \\
\hline & a-CIM 440,620 & a-CIM ${ }_{440,675}$ & a-CIM ${ }_{620,675}$ & a-CIM $440,620,675$ & b-CIM ${ }_{440,675}$ \\
\hline RMSE & 7.7577 & 15.6413 & 10.5276 & 6.8726 & 38.2312 \\
\hline$R M S E_{\text {rel }}$ & 0.2860 & 0.7737 & 0.3401 & 0.2877 & 1.5628 \\
\hline$M N B$ & -0.0219 & 0.0760 & 0.0202 & -0.0602 & 0.3292 \\
\hline NRMS & 0.2913 & 0.7865 & 0.3468 & 0.2874 & 1.5606 \\
\hline \multirow{2}{*}{$\begin{array}{l}\text { 评价 } \\
\text { 指标 }\end{array}$} & \multicolumn{5}{|c|}{ 三藻混合实验 } \\
\hline & ${ }^{a-C I M}{ }_{440,620}$ & a-CIM ${ }_{440,675}$ & ${ }^{\mathrm{a}-\mathrm{CIM}_{620,675}}$ & a-CIM $440,620,675$ & b-CIM $_{440,675}$ \\
\hline RMSE & 21.1552 & 34.0850 & 23.0396 & 13.7448 & 17.4992 \\
\hline$R M S E_{\text {rel }}$ & 3.3130 & 1.3905 & 1.2703 & 0.6345 & 2.9911 \\
\hline$M N B$ & 0.5031 & 0.0917 & 0.0813 & -0.2141 & 0.7507 \\
\hline NRMS & 3.3369 & 1.4139 & 1.2919 & 0.6086 & 2.9505 \\
\hline
\end{tabular}
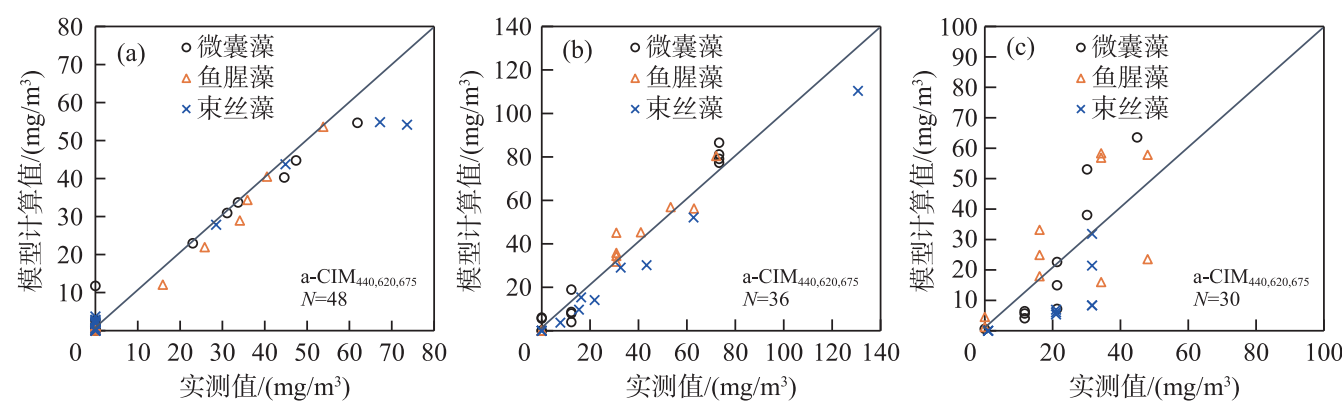

图 3 基于纯藻 (a)、双藻 (b) 和三藻 (c) 实验数据的 a-CIM $440,620,675$ 模型

3 种蓝藻的叶绿素 $a$ 浓度计算值与监测值对比

Fig.3 Chlorophyll- $a$ concentration comparison of model predictions and measurements based on single cyanobacteria specie (a), bi-cyanobacteria-specie (b) and tri-cyanobacteria-specie (c) experiments 
2.1.3 三藻实验结果 3 种蓝藻混合实验验证 5 种定量识别模型发现, 由于 3 种水华蓝藻在吸收和散射上的 累积叠加, 吸收、散射数据的复杂性进一步降低了模型的定量识别能力, 总体上, 基于吸收的三波段模型 $\mathrm{a}-\mathrm{CIM}_{440,620,675}$ 具有相对较好的定量识别能力. 图 3c 是该模型定量识别值和监测值的对比, 从图中看出 3 种蓝 藻的结果均较为分散地分布在 $1: 1$ 线附近. 由于在三藻混合实验中, 3 种蓝藻的 Chl. $a$ 浓度以间隔 $<20 \mathrm{mg} / \mathrm{m}^{3}$ 的 浓度依次递增, 没有明显的主导蓝藻, 因此每种主要水华蓝藻的吸收、散射特征不突出, 从而降低了模型的 定量识别能力.

\section{2 巢湖主要水华蓝藻类群的时空分布特征}

蓝藻占巢湖藻种的绝对主导地位, 主要由微囊藻、鱼腥藻、束丝藻这 3 种藻类构成 ${ }^{[6-7]}$, 因此本研究结合 实测 Chl. $a$ 总浓度和各特征波段的吸收系数将本研究提出的水华蓝藻定量识别模型应用于巢湖, 可以获取 巢湖 3 种水华蓝藻的时空分布特征, 同时也能初步验证该模型在巢湖的适用性.

本研究的水华蓝藻类群定量识别模型建立在室内光学控制实验基础之上, 蓝藻的吸收系数是在实验室 通过 AC-S 仪器实测获得. 巢湖野外样品的浮游植物总吸收系数采用 QFT-分光光度计方法测得. 两种方法 之间存在着系统误差. 周雯 ${ }^{[37]}$ 的研究发现, QFT-分光光度计方法测得的吸收系数与色素浓度线性关系较 差, 实验测量引人的误差也较大, 且其测得的吸收系数平均值高于 AC-S 测量吸收系数. 在野外条件下, 受水 体中无机悬浮物和黄色物质的影响, AC-S 只能获取水体的总吸收系数, 无法直接获取浮游植物总吸收.

为了初步判断该模型在巢湖的适用性, 将本研究水华蓝藻类群定量识别模型应用于巢湖, 需要做以下 假定: (1) 假定 AC-S 与 QFT-分光光度计方法所测的浮游植物总吸收之间的系统误差可忽略; (2) 假定巢湖 野外采样获取的 Chl. $a$ 浓度和浮游植物总吸收均来自 3 种水华蓝藻的贡献.

基于 3 个特征吸收波段的 $\mathrm{a}-\mathrm{CIM}_{440,620,67}$ 模型, 巢湖 3 种主要水华蓝藻类群的时空分布如图 4 所示. 可以 看出, 2016 年 12 月, 各监测点 3 种水华蓝藻浓度 (Chl. $a$ 浓度) 均较低, 西部监测点鱼腥藻浓度明显高于东部 湖区, 微囊藻和束丝藻浓度总体较低, 束丝藻在中部湖区的浓度略高于东部和西部湖区. 2017 年 2 月, 巢湖 各监测点几乎均以鱼腥藻为主, 全湖鱼腥藻浓度相当; 微囊藻和束丝藻浓度在所有监测点均很低; 束丝藻浓 度在东部湖区要略高于其他湖区. 2017 年 4 月,巢湖中西部各监测点的鱼腥藻浓度较 2 月份有下降, 但东部 湖区的鱼腥藻浓度较高, 且出现了鱼腥藻水华; 微囊藻浓度除在水华区域监测点稍高外, 非水华区域依然较 低; 束丝藻浓度在所有监测点都很低. 2017 年 8 月, 各监测点依然以鱼腥藻和微囊藻为主, 其中西部湖区出 现了以微囊藻为绝对优势的蓝藻水华现象, 其余监测点鱼腥藻和微囊藻浓度相近; 束丝藻浓度总体上是 4 次巡测的最低值.

总体上, 巢湖的水华蓝藻以鱼腥藻、微囊藻为主, 温度较低的季节鱼腥藻占绝对优势, 主要与内源环境 因素有关, 冬季巢湖溶解氧浓度较高且磷浓度较低, 而且温度较低, 适宜鱼腥藻生存. 微囊藻主要发生于夏 季的西部湖区, 于湖面形成水华, 是流域西北部的外源污染负荷所致. 微囊藻自身的无机碳浓缩机制和伪空 胞调节, 以及巢湖夏季 $20 \sim 34^{\circ} \mathrm{C}$ 的适宜温度, 较高的氮、磷浓度, 较高的 $\mathrm{pH}$ 值, 适宜的光照强度和时间等环 境条件, 是微囊藻水华多发生在夏季的主要原因. 由于高温对鱼腥藻 $\left(13 \sim 30^{\circ} \mathrm{C}\right)$ 、束丝藻 $\left(13 \sim 25^{\circ} \mathrm{C}\right)$ 生长代 谢有明显的抑制作用, 因此夏季鱼腥藻和束丝藻浓度总体偏低, 上述结论与马健荣等 ${ }^{[38]}$ 、刘菲菲等 ${ }^{[39]}$ 、Jiang 等 ${ }^{[6]}$ 、Zhang 等 ${ }^{[7]}$ 对巢湖蓝藻种群结构的调查结果完全一致,证实了该模型在巢湖的适用性.

\section{3 巢湖主要水华蓝藻类群的垂向分布特征}

水华蓝藻因其独特的生理结构 (伪空胞) 以及外环境水动力条件, 通过上浮或下沉来改变其垂向位置, 选择最佳的生存空间 ${ }^{[40-41}$. 因此,蓝藻垂向位置迁移造成了水体表层藻类信息难以全面、准确地表征水华蓝 藻的垂向分布特征. 本研究在已知总 Chl. $a$ 浓度和浮游植物总吸收的剖面分布的情况下,基于 3 个特征吸收 波段的 a-CIM $440,620,675$ 模型获取了 3 种水华蓝藻的垂向分布,这为科学诠释不同水华蓝藻类群的垂向分布规 律提供了重要的技术支撑.

非藻华条件下断面 $1^{\#} 、 4^{\#}$ 和 $5^{\#}$ 中 5 种水华蓝藻的垂向分布情况见图 5. 由图 5 可知, 3 个断面均以微囊 藻和鱼腥藻为主, 且均呈垂向均匀分布状态, $1^{\#}$ 和 $4^{\#}$ 断面束丝藻浓度整体偏低, $5^{\#}$ 断面水面以下 $1 \mathrm{~m}$ 范围内 主要为 $10 \mathrm{mg} / \mathrm{m}^{3}$ 左右的束丝藻. $2^{\#}$ 和 $3^{\#}$ 断面出现蓝藻水华时主导的蓝藻完全不同: $2^{\#}$ 断面主要发生的是微 囊藻藻华; $3^{\#}$ 断面则是鱼腥藻藻华 (图 6). 微囊藻自身含有气囊, 在气温和光照适宜的条件下, 通过扩大气囊 

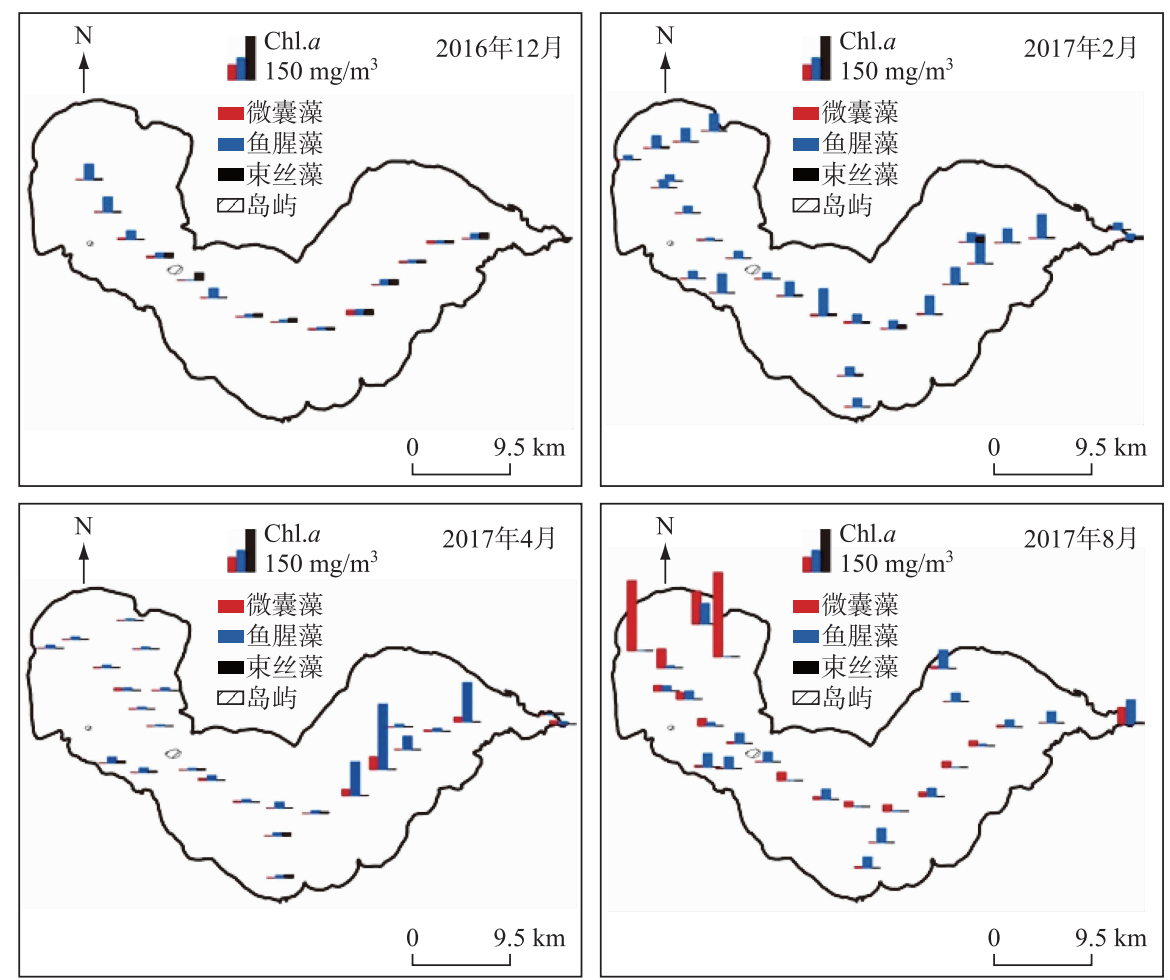

图 4 巢湖主要水华蓝藻类群的时空分布

Fig.4 Temporal and spatial distribution of main bloom-forming cyanobacteria in Lake Chaohu
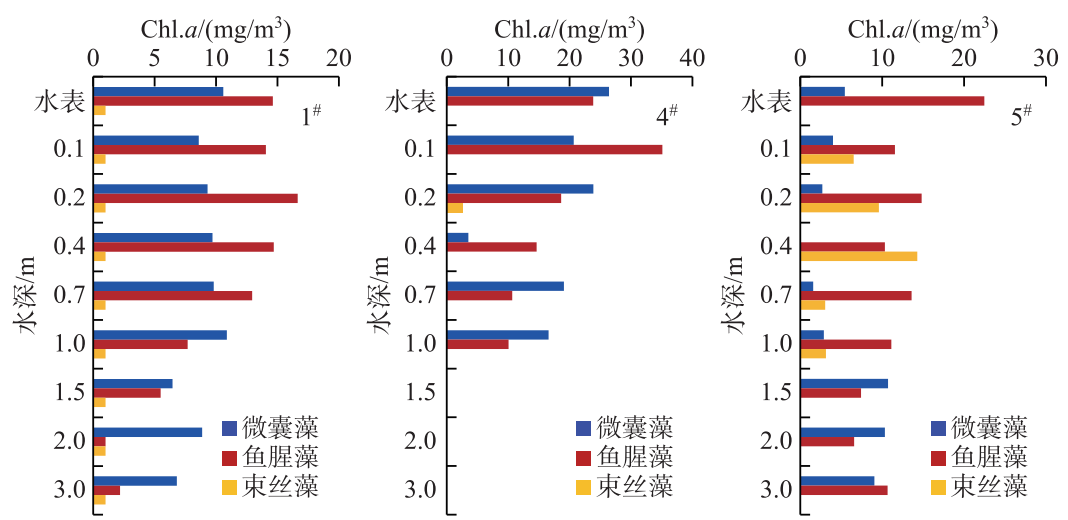

图 5 非藻华条件下 3 种主要水华蓝藻的垂向分布

Fig.5 Vertical distribution of main bloom-forming cyanobacteria at non-bloom condition

上浮至水体表层; 鱼腥藻自身并无气囊, 但是当其浓度较高、形成抱团状态时, 较大的比表面积使其更加容 易上浮至水体表面形成水华. 浓度较高 $\left(>30 \mathrm{mg} / \mathrm{m}^{3}\right)$ 的水华蓝藻主要出现在水体表面以下 $20 \mathrm{~cm}$ 范围内.

\section{3 问题与展望}

本研究基于室内光学控制实验构建了 3 种水华蓝藻的定量识别模型, 其精度还需结合大量野外实测数 据进行验证与优化; 在以水华蓝藻为主的湖泊进行野外实测时通常以数量作为衡量藻类群落结构的方式, 


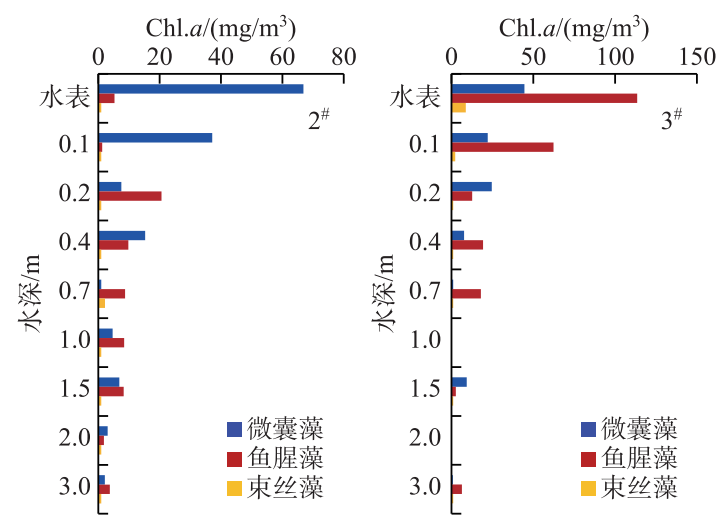

图 6 藻华条件下 3 种主要水华蓝藻的垂向分布

Fig.6 Vertical distribution of main bloom-forming cyanobacteria at bloom condition

与本研究测量以及计算的 Chl. $a$ 浓度指标具有不一致性, 难以直接进行验证. 本研究是水华蓝藻主要类群遥 感定量监测的重要尝试, 结果呈现的巢湖蓝藻类群的时空变化与传统方法得到的变化趋势一致, 可以为相 关部门准确掌握富营养化湖泊水华蓝藻的时空分布状况、科学开展湖泊水污染治理与生态修复提供重要的 决策依据.

实现富营养化湖泊水华蓝藻类群的卫星遥感监测是本项研究的最终目标. 近年来新增的海洋水色传感 器主要有可见光成像辐射仪 (VIIRS)、第二代海洋水色监视仪 (OCM-2)、地球静止海洋水色成像仪 (GOCI)、 海洋和陆地颜色仪 (OLCI) 等. 其中, Sentinel-3A OLCI 的波段设置与 3 种水华蓝藻固有光学特性的特征波段 恰好匹配, 为下一步研究提供了重要的遥感数据基础. 但是要实现基于卫星影像开展水华蓝藻的定量识别 目标, 还需解决富营养化湖泊水体浮游植物吸收特性及其色素浓度的卫星遥感反演算法研究. 受人类影响 最强烈的富营养化湖泊, 属于典型的 II 类水体, 其光学成因复杂, 传统大洋 I 类水体的大气校正算法、色素 浓度反演算法和颗粒物固有光学特性反演算法的适用性不强. 因此, 基于卫星影像实现富营养化湖泊主要 水华蓝藻空间分布的遥感定量反演,还需水色遥感相关理论研究的不断发展和进步,任重而道远.

\section{4 参考文献}

[ 1 ] Ministry of Environmental Protection, PRC ed. Bulletin of the state of the environment of China. Beijing: China Environmental Science Press, 2017. [中华人民共和国环境保护部. 中国环境状况公报. 北京: 中国环境科学出版 社, 2017.]

[ 2 ] Reynolds CS, Walsby AE. Water-blooms. Biological Reviews, 1975, 50(4) : 437-481. DOI : 10.1111/j.1469-185x.1975. tb01060.x.

[ 3 ] Reynolds CS ed. Cyanobacterial water-blooms. Advances in botanical research. Amsterdam: Elsevier, 1987: 67-143. DOI: 10.1016/s0065-2296( 08) 60341-9.

[ 4 ] Wu QL, Xie P, Yang LY et al. Studies on the mechanism of ecological disaster of harmful algal blooms in shallow lakes. Advantages Earth Science, 2008, 23(11): 1115-1123. [吴庆龙, 谢平, 杨柳燕等. 湖泊蓝藻水华生态灾害形成机理 及防治的基础研究. 地球科学进展, 2008, 23(11): 1115-1123.]

[ 5 ] Liu Y. Studies on the diversity, spatial-temporal distribution, and physiological characters of Anabaena flos-aquae in Lake Taihu [Dissertation]. Beijing: University of Chinese Academy of Sciences, 2013. [刘洋. 太湖水华鱼腥藻的多样性、时 空分布及生理特性研究 [学位论文]. 北京: 中国科学院大学, 2013.]

[ 6 ] Jiang YJ, He W, Liu WX et al. The seasonal and spatial variations of phytoplankton community and their correlation with environmental factors in a large eutrophic Chinese lake (Lake Chaohu). Ecological Indicators, 2014, 40: 58-67. DOI: 10. 1016/j. ecolind.2014.01.006.

[ 7 ] Zhang M, Zhang YC, Yang Z et al. Spatial and seasonal shifts in bloom-forming cyanobacteria in Lake Chaohu: Patterns 
and driving factors. Phycological Research, 2016, 64(1): 44-55. DOI: 10.1111/pre.12112.

[ 8 ] Pech-Pacheco JL, Alvarez-Borrego J. Optical-digital system applied to the identification of five phytoplankton species. Marine Biology, 1998, 132(3) : 357-365. DOI: 10.1007/s002270050402.

[ 9 ] Qiao XY. Automatic recognition method of microscopic image of harmful algae. Journal of Shandong University: Engineering Science, 2016, 46(3) : 1-6. [乔小燕. 赤潮藻显微图像自动识别方法. 山东大学学报: 工学版, 2016, 46(3): 1-6. ]

[10] Chen Y, Liu GQ, Li WB et al. Comparison of three extraction methods for DNA from Chlorella spp. Plant Physiology Communications, 2001, 37(3): 242-244. [陈颖, 刘根齐, 李文涁等. 3 种小球藻 DNA 提取方法的比较. 植物生理学通 讯, 2001, 37(3): 242-244.]

[11] MacKey MD, MacKey DJ, Higgins HW et al. CHEMTAX User's Manual: A program for estimating class abundances from chemical markers-Application to HPLC measurements of phytoplankton pigments. Marine Ecology Progress, 1996,144 (1) : 265-283. DOI: $10.3354 /$ meps 144265 .

[12] Lewitus AJ, White DL, Tymowski RG et al. Adapting the CHEMTAX method for assessing phytoplankton taxonomic composition in Southeastern US estuaries. Estuaries, 2005, 28(1) : 160-172. DOI: 10.1007/bf02732761.

[13] Hu XP, Su RG, Zhang CS et al. Fluorescence discrimination technology for the red tide algae by spectra similarity index. Chinese Journal of Lasers, 2008, 35(1) : 115-119. [ 胡序朋, 苏荣国, 张传松等. 基于光谱相似性指数的赤潮藻苂光 识别技术. 中国激光, 2008, 35(1): 115-119.]

[14] Zhang SS, Su RG, Duan YL. Research on the 3D discrete fluorescence spectrum technique for differentiation of phytoplankton population. Spectroscopy and Spectral Analysis, 2011, 31(3): 732-736. [张珊珊, 苏荣国, 段亚丽等. 浮游植 物群落组成的离散三维苂光光谱识别测定技术. 光谱学与光谱分析, 2011, 31(3): 732-736.]

[15] Moberg L, Karlberg B, Sørensen K et al. Assessment of phytoplankton class abundance using absorption spectra and chemometrics. Talanta, 2002, 56(1) : 153-160. DOI: 10.1016/S0039-9140(01) 00555-0.

[16] Lu L. Study on fluorescence spectra for identifying phytoplankton community [Dissertation]. Qingdao: Scean University of China, 2007. [卢璐. 浮游植物群落组成苂光识别测定方法研究 [学位论文]. 青岛: 中国海洋大学, 2007.]

[17] Mahoney KL. Backscattering of light by Karenia brevis and implication for optical detection and monitoring [Dissertation]. Hattiesburg: University of Southern Mississippi, 2003.

[18] Martínez-Guijarro R, Romero I, Pachés M et al. Determination of phytoplankton composition using absorption spectra. Talanta, 2009, 78(3) : 814-819. DOI: 10.1016/j.talanta.2008.12.073.

[19] Huang CC, Li YM, Sun DY. Identification of algea using photosensitive pigment absorption signals in Tiahu Lake. Acta Hydrobiologica Sinica, 2010, 34(3) : 582-590. [黄昌春, 李云梅, 孙德勇等. 基于感光色素吸收信号的太湖藻类识 别. 水生生物学报, 2010, 34(3): 582-590.]

[20] Boddy L, Morris CW, Wilkins MF et al. Identification of 72 phytoplankton species by radial basis function neural network analysis of flow cytometric data. Marine Ecology Progress Series, 2000, 195(1) : 47-59. DOI: 10.3354/meps195047.

[21] Ma WQ. Study on biological optical properties remote sensing identification of Microcysis [Dissertation]. Nanjing: Nanjing Normal University, 2012. [马万泉. 微囊藻生物光学特性与遥感识别研究 [ 学位论文]. 南京: 南京师范大 学, 2012.]

[22] Dai HL. The scattering characteristics and theoretical analog of the primary algae species in freshwater lakes [Dissertation]. Nanjing: Nanjing Normal University, 2013. [ 戴红亮. 淡水湖泊主要藻种的散射特性和理论模拟研究 [学位论文]. 南京: 南京师范大学, 2013.]

[23] Lv H, Dai HL, Li YM et al. simulating the light absorption and scattering properties of Microsystis aeruginosa using a twolayered spherical geometry. Acta Optica Sinica, 2013, 12: 319-329. [吕恒, 戴红亮, 李云梅等. 铜绿微囊藻吸收和散 射特性两层球形模型模拟. 光学学报, 2013, 12: 319-329.]

[24] Lv H, Wang Y, Li XJ et al. Scattering and backscattering properties of main fresh water alga species. Bulletin of Botany, 2014, 49 (5): 578-586. [吕恒, 王瑜, 李晓俊等. 内陆湖泊主要藻种散射特性. 植物学报, 2014, 49(5): 578-586.]

[25] Wang Y. Study on estimation of Micocystis aeruginosa proportion in Taihu Lake based on optical absorption spectra[Dissertation]. Nanjing: Nanjing Normal University, 2014. [王瑜. 基于吸收光谱的太湖铜绿微囊藻比例估算研究 [学位论 文]. 南京: 南京师范大学, 2014.] 
[26] Cui TW, Zhang J, Ma Y et al. Study of red tide spectral characteristics and its mechanism. Spectroscopy and Spectral Anal$y s i s, 2006,26(5)$ : 884-886. [崔廷伟, 张杰, 马毅等. 赤潮光谱特征及其形成机制. 光谱学与光谱分析, 2006,26 (5) : 884-886.]

[27] Lee Z, Carder KL, Du KP. Effects of molecular and particle scatterings on the model parameter for remote-sensing reflectance. Applied Optics, 2004, 43(25) : 4957-4964. DOI: 10.1364/ao.43.004957.

[28] Hunter PD, Tyler AN, Présing M et al. Spectral discrimination of phytoplankton colour groups: The effect of suspended particulate matter and sensor spectral resolution. Remote Sensing of Environment, 2008, 112(4) : 1527-1544. DOI: 10. 1016/j.rse.2007.08.003.

[29] Zhang YX, Zhang YC, Zhou W et al. Inherent optical properties of typical cyanobacteria in eutrophic lakes. J Lake Sci, 2018，30(6) : 1681-1692. DOI: 10.18307/2018.0620. [张壹萱, 张玉超, 周雯等. 富营养化湖泊典型水华蓝藻的固 有光学特性. 湖泊科学, 2018, 30(6): 1681-1692.]

[30] Chen X, Yang XD, Dong XH et al. Environmental changes in Chaohu Lake (southeast, China) since the mid 20th century: The interactive impacts of nutrients, hydrology and climate. Limnologica, 2013, 43(1) : 10-17. DOI: 10.1016/j.limno.2012.03.002.

[31] Mueller JL, Lange RE. Bio-optical provinces of the Northeast Pacific Ocean: A provisional analysis. Limnology and Oceanography, 1989, 34(8) : 1572-1586. DOI: 10.4319/lo.1989.34.8.1572.

[32] Duan HT, Ma RH, Hu CM. Evaluation of remote sensing algorithms for cyanobacterial pigment retrievals during spring bloom formation in several lakes of East China. Remote Sensing of Environment, 2012, 126: 126-135. DOI: 10.1016/j.rse. 2012.08.011.

[33] Moses WJ, Gitelson AA, Berdnikov SV et al. Estimation of chlorophyll-a concentration in case II waters using MODIS and MERIS data-successes and challenges. Environmental Research Letters, 2009, 4(4) : 045005. DOI: 10. 1088/17489326/4/4/045005.

[34] Simis SGH, Ruiz-Verdú A, Domínguez-Gómez JA et al. Influence of phytoplankton pigment composition on remote sensing of cyanobacterial biomass. Remote Sensing of Environment, 2007, 106(4) : 414-427. DOI: 10.1016/j.rse.2006.09.008.

[35] Mobley CD. Estimation of the remote-sensing reflectance from above-surface measurements. Applied Optics, 1999, 38 (36) : 7442-7455. DOI: 10.1364/ao.38.007442.

[36] Mentaschi L, Besio G, Cassola F et al. Problems in RMSE-based wave model validations. Ocean Modelling, 2013,72 : 53-58. DOI: 10.1016/j.ocemod.2013.08.003.

[37] Zhou W. Theoretical simulation of phytoplankton light scattering characteristics [Dissertation]. Guangzhou: South China Sea Institute of Oceanology, Chinese Academy of Sciences, 2008. [周雯. 浮游植物光散射特性理论模拟 [学位论文]. 广州: 中国科学院南海海洋研究所, 2008.]

[38] Ma JR, Deng J, Qin BQ et al. Progress and prospects on cyanobacteria bloom-forming mechanism in lakes. Acta Ecologica Sinica, 2013, 33(10) : 3020-3030. [马健荣, 邓建明, 秦伯强等. 湖泊蓝藻水华发生机理研究进展. 生态学报, $2013, \mathbf{3 3}(10): 3020-3030$.]

[39] Liu FF, Feng MH, Shang LX et al. Effects of temperature on the growth and generation of extracellular organic matter of Microcystis aeruginosa and Anabaena sp. J Lake Sci, 2014, 26(5) : 780-788. DOI: 10.18307/2014.0517. [刘菲菲, 冯 慕华, 尚丽霞等. 温度对铜绿微囊藻 (Microcystis aeruginosa) 和鱼腥藻 (Anabaena sp.) 生长及胞外有机物产生的影 响. 湖泊科学, 2014, 26(5) : 780-788.

[40] Walsby AE. Gas vesicles. Microbiological Reviews, 1994, 58(1) : 94-144. DOI: 10.1128/mmbr.58.1.94-144.1994.

[41] Kong FX, Song LR eds. Study on the formation process and environmental characteristics of cyanobacteria bloom. Beijing: Science Press, 2011. [ 孔繁翔, 宋立荣. 蓝藻水华形成过程及其环境特征研究. 北京: 科学出版社, 2011.] 